\title{
Effect of erythropoietin levels on mortality in old age: the Leiden 85-plus Study
}

\author{
Wendy P.J. den Elzen MSc, Jorien M. Willems MD, Rudi G.J. Westendorp MD PhD, \\ Anton J.M. de Craen PhD, Gerard Jan Blauw MD PhD, Luigi Ferrucci MD PhD, \\ Willem J.J. Assendelft MD PhD, Jacobijn Gussekloo MD PhD
}

Previously published at www.cmaj.ca

\section{ABSTRACT}

Background: The production of erythropoietin is triggered by impaired oxygen delivery to the kidney, either because of anemia or hypoxemia. High erythropoietin levels have been shown to predict the risk of death among patients with chronic heart failure. We investigated the prognostic value of elevated erythropoietin levels on mortality among very elderly people in the general population.

Methods: The Leiden 85-plus Study is a population-based prospective follow-up study involving 599 people aged 85 years in Leiden, the Netherlands, enrolled between September 1997 and September 1999. Erythropoietin levels were determined at age 86 . For this analysis, we included 428 participants with a creatinine clearance of at least $30 \mathrm{~mL} / \mathrm{min}$. Mortality data, recorded until Feb. 1, 2008, were obtained from the municipal registry.

Results: During follow-up, 324 (75.7\%) participants died. Compared with participants whose erythropoietin levels were in the lowest tertile (reference group), those whose levels were in the middle tertile had a $25 \%$ increased risk of death (hazard ratio [HR] 1.25, 95\% confidence interval [CI] 0.951.64), and those whose levels were in the highest tertile had a $73 \%$ increased risk (HR 1.73, 95\% Cl 1.32-2.26) ( $p$ value for trend $<0.01$ ). The association between erythropoietin levels and mortality remained largely unchanged after we adjusted for sex, creatinine clearance, hemoglobin level, comorbidity, smoking status and C-reactive protein level, and was similar for deaths from cardiovascular and noncardiovascular causes.

Interpretation: Among people aged 85 years and older, elevated erythropoietin levels were associated with an in creased risk of death, independent of hemoglobin levels.

$\mathrm{D}$ ecreased oxygen availability in the kidney triggers the peritubular capillary lining cells within the kidney to produce erythropoietin - the principal regulator of red blood cell mass. ${ }^{1}$ Impaired oxygen delivery to the kidney can result from various pathophysiologic mechanisms, such as anemia, hypoperfusion due to renal arteriosclerosis, decreased renal blood flow or heart failure, and decreased oxygen saturation due to diseases such as chronic obstructive pulmonary disease. ${ }^{1-5}$
Studies involving patients with chronic heart failure have shown that high erythropoietin levels predict poor survival. ${ }^{6-11}$ However, whether this prognostic value is limited to patients with heart failure or whether it is generalizable to very elderly people regardless of specific pathologic conditions has not been studied. We conducted this study to investigate whether high levels of erythropoietin predict mortality among very elderly people in the general population.

\section{Methods}

\section{Study population}

The Leiden 85-plus Study is a population-based prospective follow-up study involving 85-year-old inhabitants of Leiden, the Netherlands. Between September 1997 and September 1999, 705 inhabitants of Leiden reached the age of 85 years and were eligible for participation in the study. Fourteen people died before enrolment; of the remaining 691, 92 refused to participate, for a response rate of $86.7 \% .^{12}$ Of the 599 participants enrolled in the study at age 85 years, 47 died before the age of 86,39 refused further participation, and 16 refused blood sampling. For six participants, their erythropoietin level could not be measured because of technical problems. Erythropoietin levels were available for 491 participants at age 86 . Because erythropoietin production is limited in patients with severe renal failure, we excluded 44 participants who had severe renal failure (defined as a Cockcroft-Gault creatinine clearance of less than $30 \mathrm{~mL} / \mathrm{min}$ ) and 19 for whom data on their creatinine clearance were not available from the analyses. We included the remaining 428 participants in our study. Participants were visited at their place of residence for face-to-face interviews,

From the Departments of Public Health and Primary Care (den Elzen Assendelft, Gussekloo) and of Gerontology and Geriatrics (Willems, Westendorp, de Craen, Blauw), Leiden University Medical Center, Leiden, the Netherlands; the Netherlands Consortium for Healthy Ageing (Westendorp), Leiden, the Netherlands; the Department of Internal Medicine (Blauw), Bronovo Hospital, The Hague, the Netherlands; and the Longitudinal Studies Section (Ferrucci), Clinical Research Branch, National Institute on Aging, National Institutes of Health, Baltimore, USA

CMAJ 2010. DOI:10.1503/cmaj.100374 
the assessment of functional tests and the collection of a venous blood sample. All participants gave their informed consent.

The Medical Ethical Committee of Leiden University Medical Center approved the study.

\section{Laboratory measurements}

Nonfasting blood samples were drawn before $11 \mathrm{am}$, and serum samples were stored at $-80^{\circ} \mathrm{C}$.

In 2008, serum erythropoietin levels were measured in one batch with the use of an enzyme immunoassay (Immulite 2500, Siemens Medical Diagnostics, Tarrytown, USA; sensitivity $1.2 \mathrm{IU} / \mathrm{L}$, coefficient of variation $<6 \%$ ). Hemoglobin, creatinine and C-reactive protein levels were determined on the day the blood sample was drawn. Hemoglobin levels were measured with the use of a fully automated system (Sysmex XE-2100, TOA Medical Electronics, Kobe, Japan). Anemia was defined according to criteria of the World Health Organization (hemoglobin level $<130 \mathrm{~g} / \mathrm{L}$ for men and $<120 \mathrm{~g} / \mathrm{L}$ for women). ${ }^{13}$ The serum creatinine concentration was measured according to the Jaffe method with the use of a fully automated Hitachi 747 system (Hitachi, Tokyo, Japan). We estimated creatinine clearances using the Cockcroft-Gault formula. ${ }^{14}$ The C-reactive protein level was used as a marker of inflammation and was measured with the use of the Hitachi 747 automated analyzer (Hitachi, Tokyo, Japan).

\section{Other clinical parameters}

Information on the presence of disease (myocardial infarction in medical history, stroke in medical history, heart failure, chronic obstructive pulmonary disease, cancer, diabetes mellitus and cognitive impairment) was obtained from general practitioners, nursing-home physicians, pharmacy records and laboratory findings. ${ }^{15}$ Diabetes mellitus was considered present if diagnosed by the primary care physician, if the nonfasting glucose level was greater than $11.0 \mathrm{mmol} / \mathrm{L}$ or if a participant was taking antidiabetic medication. The presence of severe cognitive impairment was based either on a diagnosis by the general practitioner or on a Mini-Mental State Examination score of less than 19. ${ }^{16}$ Data on the use of angiotensin-converting-enzyme inhibitors and angiotensin-receptor blockers at baseline were obtained from pharmacy records. All of the participants were interviewed about their current and former smoking habits.

\section{Mortality data}

Mortality data, recorded from the start of the study until Feb. 1, 2008, were obtained from the municipal registry. Causes of death were obtained from Statistics Netherlands, where all national death certificates are coded according to the International Classification of Diseases and Related Disorders, 10th revision. Causes of death were divided into cardiovascular causes (codes I00-I99) and noncardiovascular causes (all other codes).

Table 1: Baseline characteristics of 428 participants at age 86 years, by tertile of erythropoietin level

\begin{tabular}{|c|c|c|c|c|c|}
\hline Characteristic & $\begin{array}{c}\text { Total } \\
n=428\end{array}$ & \multicolumn{3}{|c|}{ Tertile of erythropoietin level;* no (\%) of participants } & $p$ value $\ddagger$ \\
\hline Sex, male & $142(33.2)$ & $46(32.2)$ & $45(31.5)$ & $51(35.9)$ & 0.50 \\
\hline Erythropoietin, median (IQR), IU/L & $10.4(7.8-13.8)$ & $7.2(6.3-7.8)$ & $10.4(9.5-11.3)$ & $15.7(13.8-18.4)$ & - \\
\hline Hemoglobin, median (IQR), g/L & $131(123-139)$ & $134(128-142)$ & $131(125-138)$ & $128(120-136)$ & $<0.01$ \\
\hline $\begin{array}{l}\text { Creatinine clearance, }{ }^{* *} \text { median (IQR), } \\
\mathrm{mL} / \mathrm{min}\end{array}$ & $44.3(38.9-51.8)$ & $43.7(38.5-50.5)$ & $43.8(38.1-53.1)$ & $46.3(39.6-52.7)$ & 0.04 \\
\hline C-reactive protein, median (IQR), mg/L & $3(0-7)$ & $3(0-5)$ & $3(0-7)$ & $4(1-7)$ & $<0.01$ \\
\hline Cardiovascular disease $† \dagger$ & $114(26.6)$ & $32(22.4)$ & $34(23.8)$ & $48(33.8)$ & 0.03 \\
\hline Myocardial infarction & 37 (8.6) & $8(5.6)$ & $10(7.0)$ & $19(13.4)$ & 0.02 \\
\hline Cancer & $79(18.5)$ & $24(16.8)$ & $29(20.3)$ & $26(18.3)$ & 0.72 \\
\hline Diabetes mellitus & $61(14.3)$ & $16(11.2)$ & $19(13.3)$ & $26(18.3)$ & 0.09 \\
\hline Severe cognitive impairment & $87(20.3)$ & $28(19.6)$ & $34(23.8)$ & $25(17.6)$ & 0.68 \\
\hline Former or current smoker & $199(46.5)$ & $58(40.6)$ & $72(50.3)$ & $69(48.6)$ & 0.13 \\
\hline $\begin{array}{l}\text { Use of ACE inhibitors or angiotensin- } \\
\text { receptor blockers }\end{array}$ & $59(13.8)$ & $17(11.9)$ & $15(10.5)$ & $27(19.0)$ & 0.08 \\
\hline
\end{tabular}

Note: $\mathrm{ACE}=$ angiotensin-converting enzyme, $\mathrm{IQR}=$ interquartile range.

*Lowest tertile $=3.4-8.6 \mathrm{IU} / \mathrm{L}$, middle tertile $=8.7-12.3 \mathrm{IU} / \mathrm{L}$, highest tertile $=12.4-103.0 \mathrm{IU} / \mathrm{L}$.

tUnless stated otherwise.

$\ddagger p$ values were obtained by the $\chi^{2}$ test (linear-linear, 1 degree of freedom) for categorical variables and the Jonkheere-Terpstra test for continuous variables

§World Health Organization criteria: hemoglobin level $<130 \mathrm{~g} / \mathrm{L}$ for men and hemoglobin level $<120 \mathrm{~g} / \mathrm{L}$ for women.

**Determined using the Cockcroft-Gault formula.

t†Includes stroke, myocardial infarction and heart failure. 


\section{Statistical analysis}

Participants were grouped according to the tertile of erythropoietin level: low (3.4-8.6 IU/L), middle (8.7-12.3 IU/L) and high (12.4-103.0 IU/L). Differences in characteristics between participants in the three tertiles were tested with the $\chi^{2}$ test (linear-linear, one degree of freedom) for categorical variables and the Jonkheere-Terpstra test for continuous variables.

To investigate the association between erythropoietin level and risk of death, we used Kaplan-Meier plots and Cox proportional hazard models. The Kaplan-Meier plots were truncated at eight years of follow-up because of small numbers at the end of follow-up.

We constructed seven subsequent Cox proportional hazard models, each model an extension of the previous one. In the first model, we estimated the crude effect of erythropoietin level on mortality. The second model was an extension of the first by adjusting for sex. In subsequent models, we added creatinine clearance (model 3), hemoglobin level (model 4), the presence of disease (myocardial infarction, stroke, heart failure, chronic obstructive pulmonary disease, cancer, diabetes mellitus and severe cognitive impairment; model 5), smoking status (model 6) and C-reactive protein level (model 7). Erythropoietin tertile, myocardial infarction, stroke, heart failure, chronic obstructive pulmonary disease, cancer, diabetes, severe cognitive impairment and smoking status were entered as categorical variables; creatinine clearance, hemoglobin level and C-reactive protein level were entered as continuous variables. To obtain a $p$ value for trend, we included the variable for erythropoietin tertile in the model as a continuous variable.

Additional analyses were performed according to whether participants had anemia at the age of 86 . In these analyses, participants with and without anemia were classified according to strata-specific erythropoietin tertiles.

\section{Results}

The characteristics of the 428 participants at age 86 years are summarized in Table 1 . In brief, $33.2 \%$ of the study population was male. The median erythropoietin level was 10.4 (interquartile range [IQR] 7.8-13.8, range 3.4-103.0) IU/L. Anemia was present in 85 participants (19.9\%), cardiovascular disease in $114(26.6 \%)$ and chronic obstructive pulmonary disease in $41(9.6 \%)$. Participants in the highest erythropoietin tertile had lower hemoglobin levels and higher creatinine clearance and C-reactive protein levels than participants in the middle and lowest tertiles. The prevalence of anemia and cardiovascular disease was highest among participants in the highest erythropoietin tertile.

During follow-up, 324 (75.7\%) participants died: 98 $(68.5 \%)$ in the lowest erythropoietin tertile, $108(75.5 \%)$ in the middle tertile and $118(83.1 \%)$ in the highest tertile. Causes of death were available for 319 participants: 115 (36.1\%) died of cardiovascular causes and 204 (63.9\%) of noncardiovascular causes. Participants in the highest erythropoietin tertile had the highest all-cause mortality risk (Figure 1, log-rank test [2 degrees of freedom], $p<0.01)$. When compared with participants in the lowest erythropoietin tertile (reference group), those in the middle tertile had a hazard ratio (HR) for death of
1.25 (95\% confidence interval [CI] 0.95-1.64) and those in the highest tertile an HR of 1.73 (95\% CI 1.32-2.26) ( $p$ for trend $<0.01$, Table 2). These results did not change after we adjusted the analysis for sex, creatinine clearance, hemoglobin level, comorbidity, smoking status and C-reactive protein level (middle tertile, adjusted HR 1.07, 95\% CI 0.80-1.44; highest tertile, adjusted HR 1.69, 95\% CI 1.26-2.26; $p$ for trend $<0.01$ ). Similar results were obtained when we excluded participants who died during the first year of follow-up (middle tertile, adjusted HR 1.13, 95\% CI 0.83-1.55; highest tertile, adjusted HR 1.76, 95\% CI 1.30-2.39; $p$ for trend $<0.01$ ) or when we excluded participants who were using angiotensin-converting-enzyme inhibitors and angiotensin-receptor blockers (middle tertile, adjusted HR 1.06, 95\% CI 0.77-1.46; highest tertile, adjusted HR 1.72, 95\% CI 1.24-2.39; $p$ for trend < 0.01).

Figure 2 shows all-cause and cause-specific mortality from age 86 onward by baseline erythropoietin tertile, adjusted for sex, creatinine clearance, hemoglobin level, comorbidity, smoking status and C-reactive protein level. The association between erythropoietin and mortality was similar for deaths from cardiovascular causes (middle tertile, adjusted HR 1.24, 95\% CI 0.75-2.04; highest tertile, adjusted HR 1.79, 95\% CI $1.09-2.92 ; p$ for trend $=0.02$ ) and for deaths from noncardiovascular causes (middle tertile, adjusted HR 0.96, 95\% CI 0.66-1.39; highest tertile, adjusted HR 1.58, 95\% CI 1.092.29; $p$ for trend $=0.02$ ).

Overall, 57 participants died of cancer: $15(10.5 \%)$ in the lowest erythropoietin tertile, $18(12.6 \%)$ in the middle tertile

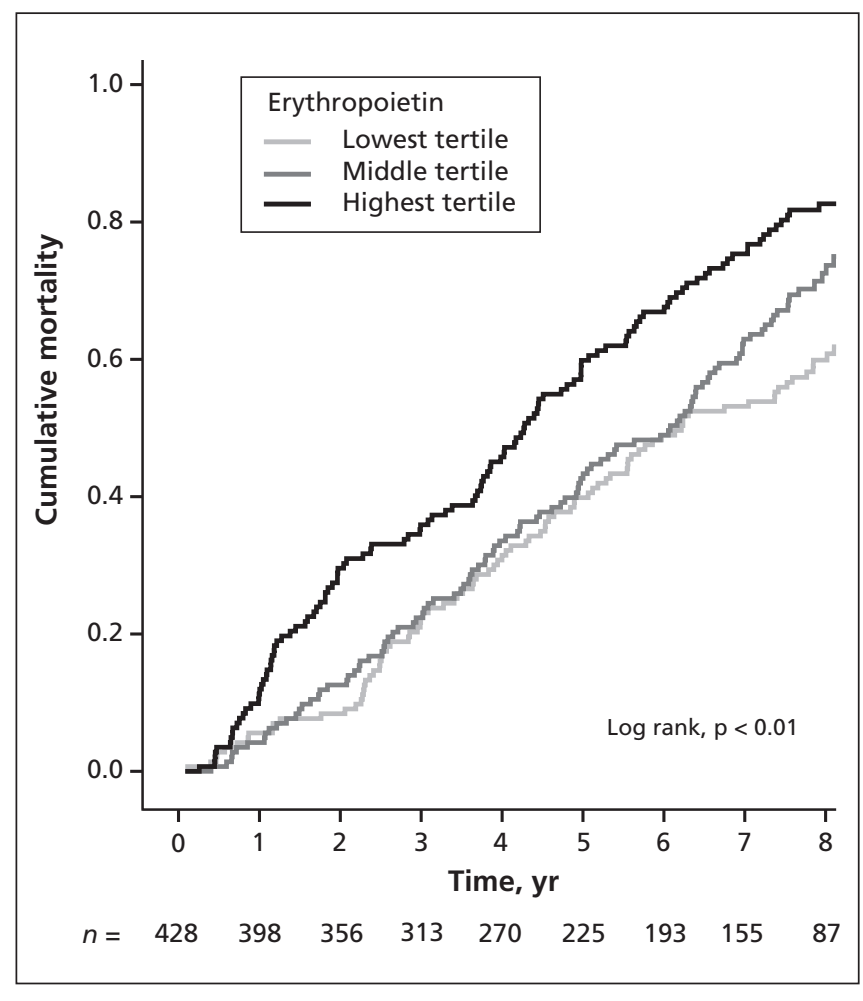

Figure 1: Cumulative all-cause mortality among 428 participants aged 86 at baseline, by tertile of erythropoietin level at baseline (lowest $=3.4-8.6 \mathrm{IU} / \mathrm{L}$, middle $=8.7-12.3 \mathrm{IU} / \mathrm{L}$, highest $=12.4-$ $103.0 \mathrm{IU} / \mathrm{L})$. 
and $24(16.9 \%)$ in the highest tertile. When compared with participants in the lowest tertile, those in the middle tertile had an adjusted HR for death from cancer of 0.92 (95\% CI 0.44-1.93) and those in the highest tertile had an adjusted HR of 1.84 (95\% CI 0.88-3.85) ( $p$ for trend $=0.10$ ).

The results of the stratified Cox proportional hazard models by presence or absence of anemia are presented in Table 3. In both groups, higher baseline erythropoietin levels were associated with an increased risk of death from all causes. Among participants without anemia $(n=343)$, high erythropoietin levels remained associated with an increased risk of death from all causes after adjustment for sex, creatinine clearance, hemoglobin level, comorbidity, smoking status and C-reactive protein level (middle erythropoietin tertile, adjusted HR 1.19,
95\% CI 0.86-1.67; highest tertile, adjusted HR 1.52, 95\% CI $1.09-2.11 ; p$ for trend $=0.01)$. Among participants who had anemia $(n=85)$, similar HRs were found after adjustment for sex, creatinine clearance, hemoglobin level, comorbidity, smoking status and C-reactive protein level; however, the trend was no longer statistically significant (middle tertile, adjusted HR 1.16, 95\% CI 0.61-2.22; highest tertile, adjusted HR 1.84, 95\% CI 0.73-4.61; $p$ for trend $=0.24$ ).

\section{Interpretation}

In this population-based sample of very elderly people, we found that high erythropoietin levels were associated with increased risk of death, independent of creatinine clearance,

Table 2: Risk of all-cause mortality among participants, by tertile of erythropoietin level at baseline

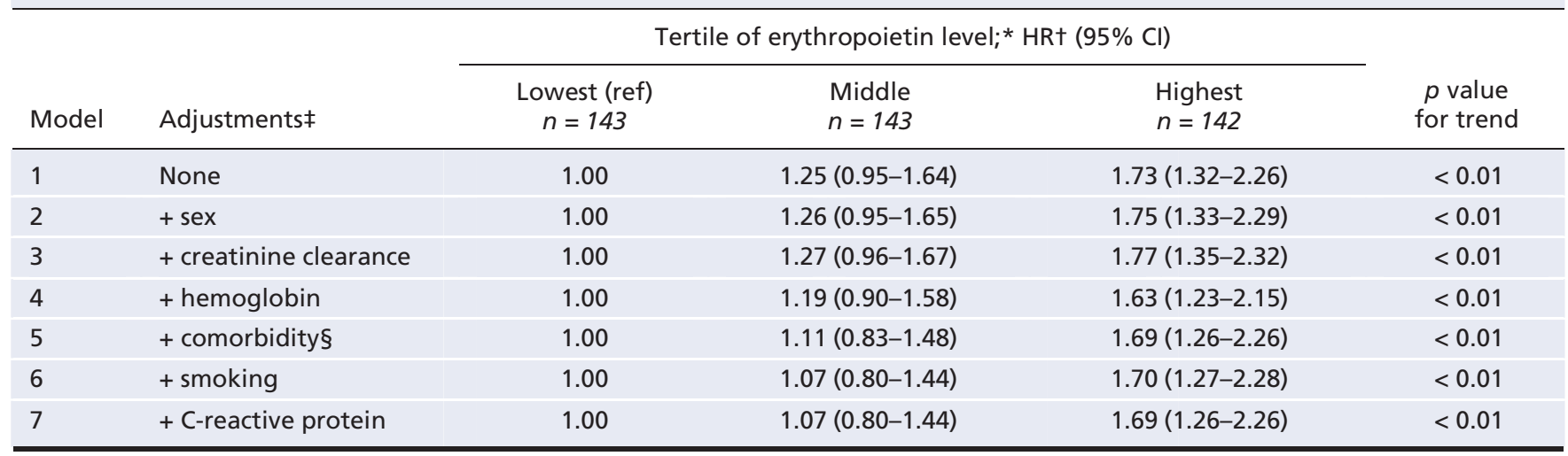

Note: $\mathrm{Cl}=$ confidence interval, $\mathrm{HR}=$ hazard ratio, ref = reference group.

* Lowest tertile = 3.4-8.6 IU/L, middle tertile = 8.7-12.3 IU/L, highest tertile $=12.4-103.0 \mathrm{IU} / \mathrm{L}$.

tHRs were estimated using Cox proportional hazard models.

¥Seven subsequent Cox proportional hazard models were performed, each an extension of the previous model.

§Includes myocardial infarction, stroke, heart failure, chronic obstructive pulmonary disease, cancer, diabetes mellitus and severe cognitive impairment.

Table 3: Risk of death among participants, by tertile of erythropoietin level and anemia status at baseline

Tertile of erythropoietin level*

\begin{tabular}{|c|c|c|c|c|c|c|c|c|c|}
\hline Model & Adjustments§ & \multicolumn{3}{|c|}{ No anemia; HRt $(95 \% \mathrm{Cl})$} & $\begin{array}{l}p \text { value } \\
\text { for trend }\end{array}$ & \multicolumn{3}{|c|}{ Anemia; $\neq \mathrm{HR} †(95 \% \mathrm{Cl})$} & $\begin{array}{l}p \text { value } \\
\text { for trend }\end{array}$ \\
\hline 1 & None & 1.00 & $1.40(1.03-1.91)$ & $1.55(1.14-2.11)$ & $<0.01$ & 1.00 & $1.27(0.71-2.28)$ & $2.03(1.16-3.56)$ & 0.02 \\
\hline 2 & $+\operatorname{sex}$ & 1.00 & $1.42(1.05-1.94)$ & $1.61(1.18-2.19)$ & $<0.01$ & 1.00 & $1.15(0.64-2.10)$ & $1.91(1.08-3.36)$ & 0.03 \\
\hline 4 & + hemoglobin & 1.00 & $1.42(1.04-1.94)$ & $1.60(1.17-2.19)$ & $<0.01$ & 1.00 & $1.19(0.65-2.17)$ & $1.62(0.84-3.15)$ & 0.16 \\
\hline 5 & + comorbidity** & 1.00 & $1.24(0.89-1.72)$ & $1.51(1.09-2.10)$ & 0.01 & 1.00 & $1.09(0.58-2.08)$ & $1.80(0.77-4.22)$ & 0.23 \\
\hline 6 & + smoking & 1.00 & $1.20(0.86-1.67)$ & $1.52(1.09-2.10)$ & 0.01 & 1.00 & $1.16(0.60-2.22)$ & $1.98(0.79-4.93)$ & 0.19 \\
\hline 7 & $\begin{array}{l}+ \text { C-reactive } \\
\text { protein }\end{array}$ & 1.00 & $1.19(0.86-1.67)$ & $1.52(1.09-2.11)$ & 0.01 & 1.00 & $1.16(0.61-2.22)$ & $1.84(0.73-4.61)$ & 0.24 \\
\hline
\end{tabular}

Note: $\mathrm{Cl}=$ confidence interval, $\mathrm{HR}=$ hazard ratio, ref = reference group.

*For participants without anemia: lowest tertile $=3.4-8.2 \mathrm{IU} / \mathrm{L}$, middle tertile $=8.3-11.7 \mathrm{IU} / \mathrm{L}$, highest tertile $=11.8-30.5 \mathrm{IU} / \mathrm{L}$. For participants with anemia: lowest tertile $=5.7-10.6 \mathrm{IU} / \mathrm{L}$, middle tertile $=10.7-14.8 \mathrm{IU} / \mathrm{L}$, highest tertile $=15.0-103.0 \mathrm{IU} / \mathrm{L}$.

†Hazard ratios were estimated using Cox proportional hazard models.

$\ddagger$ World Health Organization criteria: hemoglobin level $<130 \mathrm{~g} / \mathrm{L}$ for men and $<120 \mathrm{~g} / \mathrm{L}$ for women.

§Seven subsequent Cox proportional hazard models were performed, each an extension of the previous model.

**Includes myocardial infarction, stroke, heart failure, chronic obstructive pulmonary disease, cancer, diabetes mellitus and severe cognitive impairment. 
hemoglobin level, presence of comorbidities, smoking status and circulating markers of inflammation. The association between erythropoietin and mortality was similar for deaths from cardiovascular causes and deaths from noncardiovascular causes.

Our results build on evidence from studies involving patients with chronic heart failure..$^{6-11}$ A number of potential physiologic mechanisms may underlie the association between elevated erythropoietin and increased mortality independent of hemoglobin level. The erythropoietin level may be a biomarker of general hypoxia. Patients with sleep apnea syndrome have been found to have high erythropoietin levels, which usually return to normal after effective continuous positive airway pressure therapy. ${ }^{17}$ The erythropoietin level is also increased after acute myocardial infarction and is thought to protect against the adverse effects of ischemia and hypoxia through reduction of the infarct size, inhibition of apoptosis and promotion of new vessel formation. ${ }^{18}$ Elevated erythropoietin levels could thus be a physiologic response to a chronically increased hypoxic stimulus due to yet undiagnosed (subclinical) disease. ${ }^{19,20}$ In addition, elevated erythropoietin levels may be compensating for removal of erythrocytes from the blood, either because of hemolysis or blood loss..$^{19,20}$ Each of these hypotheses should prompt clinicians to examine very elderly patients who have elevated erythropoietin levels for underlying disease, especially if anemia is not present. Oxygen saturation or arterial oxygen tension measurements, unfortunately not available in our study, may shed light on the mechanisms that are at play and may help to identify the clinical and therapeutic implications of elevated erythropoietin levels in elderly patients.

High erythropoietin levels may also be a reflection of bone marrow resistance to endogenous erythropoietin caused by bone marrow senescence or bone marrow exhaustion, comparable to insulin resistance in type 2 diabetes mellitus., ${ }^{9,2,21,22}$ Over the past years, a number of unexpected nonhematopoietic functions of erythropoietin have been identified. ${ }^{23}$ Perhaps erythropoietin itself contributes to a poor outcome because of its recently established prothrombotic platelet-activating or tumour-promoting effects. ${ }^{7,23,24}$

In our study, high erythropoietin levels were associated with increased mortality, regardless of the presence of anemia. However, among participants with anemia, the association between erythropoietin and mortality was no longer statistically significant when hemoglobin levels were added into the Cox proportional hazard model. Although this result could have been due to the small number of participants with anemia, it may also suggest that different mechanisms underlie the associations between erythropoietin and mortality in very elderly patients with and without anemia.
We do not know whether elevated erythropoietin, apart from being a risk marker of increased mortality in very elderly patients, is also a causal risk factor for increased mortality. Our findings do not necessarily mean that very elderly people with renal failure, cancer or unexplained anemia should not be given recombinant erythropoietin. Such treatment has been shown to be effective in raising hemoglobin levels and improving the quality of life in patients with chronic renal disease and cancer, ${ }^{25-28}$ and in community-dwelling elderly patients with unexplained chronic anemia. ${ }^{29}$ In theory, if bone marrow resistance is the primary explanation for our findings, elevated erythropoietin levels may reflect a relative erythropoietin deficiency and indicate the need for treatment with recombinant erythropoietin. Still, recent meta-analyses of randomized trials showed that treatment with erythropoiesis-stimulating agents in patients with chronic renal disease or cancer had a negative influence on survival, ${ }^{30-32}$ which clearly emphasizes the need for further studies into the cause and effects of high erythropoietin levels in elderly patients. 


\section{Limitations and strengths}

Our study has several limitations. In our attempt to investigate the independent effect of elevated erythropoietin levels on mortality, we excluded participants with severe renal failure and adjusted the Cox proportional hazard models for renal function, hemoglobin level, comorbidity, smoking status and C-reactive protein level. Since all of these factors may have a direct influence on the production of erythropoietin, we adjusted for factors in the causal pathway that may have led to an underestimation of the true association between erythropoietin level and mortality. Other limitations of the study are its observational nature, which did not allow us to exclude the possibility of residual confounding by the presence of disease, and the lack of oxygen saturation or arterial oxygen measurements, which inhibited us from further exploring underlying physiologic mechanisms that could explain our findings. Another limitation of our study is its relatively small sample, especially of participants with anemia, which led to insufficient power to study the effect of erythropoietin on specific causes of death.

Our study has several strengths. The Leiden 85-plus Study is a prospective population-based study with a high response rate and complete nine-year follow-up data for mortality. Our sample reflects the general population of very elderly people and includes people with and without comorbidities. Our results were not affected by the use of recombinant erythropoietin treatment, since none of the participants in our study used this type of medication during the study period. In addition, instead of using clinical cut-off points for erythropoietin, which are still subject to debate, we used tertiles of erythropoietin levels, which allowed us to investigate whether the association between erythropoietin and mortality showed a biological gradient.

\section{Conclusion}

Elevated erythropoietin levels in very elderly people were associated with an increased risk of death, independent of hemoglobin level. Further studies are needed to explore the underlying pathophysiologic mechanisms and to identify the clinical relevance and therapeutic implications of elevated erythropoietin levels in this age group.

\section{This article has been peer reviewed.}

Competing interests: None declared.

Contributors: Jacobijn Gussekloo had full access to all of the data and takes responsibility for the integrity of the data and the accuracy of the analysis. Rudi Westendorp and Jacobijn Gussekloo contributed to the study concept and design and obtained funding for the study. Rudi Westendorp, Anton de Craen, Willem Assendelft and Jacobijn Gussekloo supervised the study. Rudi Westendorp, Anton de Craen, Gerard Jan Blauw and Jacobijn Gussekloo contributed to the acquisition of data. All of the authors contributed to the analysis and interpretation of the data. Wendy den Elzen, Rudi Westendorp, Anton de Craen and Jacobijn Gussekloo conducted the statistical analyses. Wendy den Elzen, Anton de Craen, Gerard Jan Blauw and Jacobijn Gussekloo provided administrative, technical or material support. Wendy den Elzen and Jacobijn Gussekloo drafted the manuscript, and all of the authors critically revised it for important intellectual content.

Funding: The Leiden 85-plus Study was funded in part by an unrestricted grant from the Dutch Ministry of Health, Welfare and Sports. This study was supported by the Intramural Research Program of the US National Institute on Aging, National Institute of Health. The sponsor had no role in the design and conduct of the study; in the collection, analysis and interpretation of the data; or in the preparation, review and approval of the manuscript.

\section{REFERENCES}

1. Adamson JW, Longo DL. Anemia and polycythemia: introduction [chapter 58]. In: Fauci AS, Braunwald E, Kasper DL, et al., editors. Harrison's principles of internal medicine. 17th ed. New York (NY): McGraw-Hill; 2008.

2. Krantz SB. Erythropoietin. Blood 1991;77:419-34.

3. Jelkmann W. Erythropoietin: structure, control of production, and function. Physiol Rev 1992;72:449-89.

4. Ebert BL, Bunn HF. Regulation of the erythropoietin gene. Blood 1999;94:1864-77.

5. Volpe M, Tritto C, Testa U, et al. Blood levels of erythropoietin in congestive heart failure and correlation with clinical, hemodynamic, and hormonal profiles. Am J Cardiol 1994;74:468-73.

6. van der Meer P, Voors AA, Lipsic E, et al. Prognostic value of plasma erythropoietin on mortality in patients with chronic heart failure. J Am Coll Cardiol 2004;44:63-7.

7. George J, Patal S, Wexler D, et al. Circulating erythropoietin levels and prognosis in patients with congestive heart failure: comparison with neurohormonal and inflammatory markers. Arch Intern Med 2005;165:1304-9.

8. Avkarogullari M, Bozkurt A, Akpinar O, et al. The relation between serum erythropoietin level and severity of disease and mortality in patients with chronic heart failure. Acta Cardiol 2008;63:297-302.

9. van der Meer P, Lok DJ, Januzzi JL, et al. Adequacy of endogenous erythropoietin levels and mortality in anaemic heart failure patients. Eur Heart J 2008;29:1510-5.

10. Belonje AM, Westenbrink BD, Voors AA, et al. Erythropoietin levels in heart failure after an acute myocardial infarction: determinants, prognostic value, and the effects of captopril versus losartan. Am Heart J 2009;157:91-6.

11. Belonje AM, Voors AA. van der Meer P, et al. Endogenous erythropoietin and outcome in heart failure. Circulation 2010;121:245-51.

12. der Wiel AB, van Exel E, de Craen AJ, et al. A high response is not essential to prevent selection bias: results from the Leiden 85-plus study. J Clin Epidemiol 2002;55:1119-25.

13. Nutritional anaemias. Report of a WHO scientific group. World Health Organ Tech Rep Ser 1968;405:5-37.

14. Cockcroft DW, Gault MH. Prediction of creatinine clearance from serum creatinine. Nephron 1976;16:31-41.

15. Bootsma-van der Wiel A, de Craen AJ, van Exel E, et al. Association between chronic diseases and disability in elderly subjects with low and high income: the Leiden 85-plus Study. Eur J Public Health 2005;15:494-7.

16. Heeren TJ, Lagaay AM, von Beek WC, et al. Reference values for the MiniMental State Examination (MMSE) in octo- and nonagenarians. J Am Geriatr Soc 1990;38:1093-6.

17. Winnicki M, Shamsuzzaman A, Lanfranchi P, et al. Erythropoietin and obstructive sleep apnea. Am J Hypertens 2004;17:783-6.

18. Lipsic E, Schoemaker RG, van der Meer P, et al. Protective effects of erythropoietin in cardiac ischemia: from bench to bedside. J Am Coll Cardiol 2006;48:2161-7.

19. Ershler WB, Sheng S, McKelvey J, et al. Serum erythropoietin and aging: a longitudinal analysis. J Am Geriatr Soc 2005;53:1360-5.

20. Price EA. Aging and erythropoiesis: current state of knowledge. Blood Cells $\mathrm{Mol}$ Dis 2008;41:158-65.

21. George J. Erythropoietin and outcome prediction in patients with heart failure: the plot thickens. Eur Heart J 2008;29:1481-2.

22. Ferrucci L, Guralnik JM, Woodman RC, et al. Proinflammatory state and circulating erythropoietin in persons with and without anemia. Am J Med 2005;118:1288.

23. Brines M, Cerami A. Erythropoietin-mediated tissue protection: reducing collateral damage from the primary injury response. J Intern Med 2008;264:405-32.

24. Smith KJ, Bleyer AJ, Little WC, et al. The cardiovascular effects of erythropoietin. Cardiovasc Res 2003;59:538-48.

25. Cody J, Daly C, Campbell M, et al. Recombinant human erythropoietin for chronic renal failure anaemia in pre-dialysis patients. Cochrane Database Syst Rev 2005; (3):CD003266.

26. Evans RW, Rader B, Manninen DL. The quality of life of hemodialysis recipients treated with recombinant human erythropoietin. Cooperative Multicenter EPO Clinical Trial Group. JAMA 1990;263:825-30.

27. Bohlius J, Wilson J, Seidenfeld J, et al. Recombinant human erythropoietins and cancer patients: updated meta-analysis of 57 studies including 9353 patients. J Natl Cancer Inst 2006;98:708-14.

28. Jones M, Schenkel B, Just J, et al. Epoetin alfa improves quality of life in patients with cancer: results of metaanalysis. Cancer 2004;101:1720-32.

29. Agnihotri P, Telfer M, Butt Z, et al. Chronic anemia and fatigue in elderly patients: results of a randomized, double-blind, placebo-controlled, crossover exploratory study with epoetin alfa. J Am Geriatr Soc 2007;55:1557-65.

30. Bohlius J, Schmidlin K, Brillant C, et al. Recombinant human erythropoiesisstimulating agents and mortality in patients with cancer: a meta-analysis of randomised trials. Lancet 2009;373:1532-42.

31. Phrommintikul A, Haas SJ, Elsik M, et al. Mortality and target haemoglobin concentrations in anaemic patients with chronic kidney disease treated with erythropoietin: a meta-analysis. Lancet 2007;369:381-8.

32. Tonelli M, Hemmelgarn B, Reiman T, et al. Benefits and harms of erythropoiesisstimulating agents for anemia related to cancer: a meta-analysis. CMAJ 2009;180: E62-E71.

Correspondence to: Wendy P.J. den Elzen, Department of Public Health and Primary Care, Leiden University Medical Center,

PO Box 9600, 2300 RC Leiden, the Netherlands;

w.p.j.den_elzen@lumc.nl 